sub-Sahara Africa: A review of NIH Fogarty-funded programs 20002012. J Emp Res Hum Res Ethics. 2014.Apr; 9(2):24-40. doi: 10.1525/ jer.2014.9.2.24.

13. Nderitu D. Kamaara E. Relevance of international collaborations in promoting sustainable development:The case of IU-Kenya Partnership. J Annals of Global History. 2019 [cited 2019 Dec 22]; 1(1): 1-12. Available from:https://www.sryahwapublications.com/annals-of-global-history/ pdf/v1-i1/2.pdf
14. Grant CJ.The promise of partnership :Perspectives from Kenya and the US. Forum for International Research in Education. 2014; 1(1):50-64.

15. Osborn D, Cutter A, Ullah F. Universal sustainable development goals: Understanding the transformational challenge for developed countries.Place unknown:Stakeholder Forum;2015 May[cited 2019 Nov 16]. Available from: https://sustainabledevelopment.un.org/content/ documents/1684SF_-_SDG_Universality_Report_-_May_2015.pdf

\title{
Minor gifts from pharmaceutical companies to doctors: A comparison between psychiatry and general medicine
}

\author{
EKATERINA AVITUV-FRENKEL, DAVID ISRAELI, AZGAD GOLD, DAVID SERFATY, RAEL D STROUS
}

\begin{abstract}
Pharmaceutical companies in countries that have communityoriented models of healthcare, unlike other countries with highly privatised healthcare systems, such as the United States, cannot legally advertise medications directly to patients. Thus, the physician is entirely responsible for choosing the right medication, and needs to take important professional and ethical concerns into consideration during this decision-making process. Pharmaceutical companies invest considerably in in marketing products to physicians. Often, this is in the form of "minor gifts" to the physician. This study examines variations in the number and type of such minor gifts present in the offices of psychiatrists and internists in various medical contexts in Israel. Our results showed that psychiatrists received more minor gifts than physicians in general hospitals. No significant differences were found between inpatient and outpatient psychiatric departments. It is important to increase awareness and highlight the impact of exposure to minor gifts as advertising products on doctors in order to avoid bias and maintain objectivity in clinical judgement regarding pharmacological management of patients.
\end{abstract}

Keywords: Pharmaceutical, gifts, ethics, physicians

\section{Introduction}

A complex and interdependent relationship exists between pharmaceutical companies and the medical system. At the

Authors: Ekaterina Avituv Frenkel (katefrenkel@gmail.com), Beer Yaakov Mental Health Center, Tel Aviv University, ISRAEL; David Israeli (david. israeli4@gmail.com), Kaplan Medical Center, Tel Aviv, ISRAEL; Azgad Gold (azgadgo@gmail.com),Beer Yaakov Mental Health Center, Tel Aviv University, ISRAEL; David Serfaty (david.serfaty@gmail.com), Maayenei Hayeshua Medical Center, Tel Aviv, ISRAEL; Rael D Strous (corresponding author raels@tauex.tau.ac.il), Maayenei Hayeshua Medical Center, Bnei Brak, ISRAEL, and Sackler Faculty of Medicine, Tel Aviv, ISRAEL.

To cite: Avituv-Frenkel E, Israeli D, Gold A, Serfaty D, Strous R D. Minor gifts from pharmaceutical companies to doctors: A comparison between psychiatry and general medicine. Indian J Med Ethics. 2020 Apr-Jun;5(2) NS: 116-9.DOI: 10.20529/IJME.2020.03

Published online on January 14,2019.

Manuscript Editor:Vijayaprasad Gopichandran

Peer Reviewers:

CIndian Journal of Medical Ethics, 2020 most basic level, pharmaceutical representatives play an important role in the medical ecosystem as they regularly update healthcare providers about the development of new drugs. Pharmaceutical companies use this opportunity to market medications to physicians and hence influence their prescription decision-making. In the US alone, the pharmaceutical industry invests approximately $\$ 15$ billion a year on material regarding their product, gifts to physicians, medication samples, excursions, honoraria and other incentives in order to encourage product prescription (1). In Israel, estimates show that pharmaceutical companies invest more than $\$ 100$ million a year in marketing drugs to medical doctors. This averages to a cost of approximately $\$ 10,000$ per doctor per year (2).

In Israel, the Ministry of Health introduced a directive in 2018 severely restricting contact between physicians and pharmaceutical representatives, and prohibiting the marketing of prescription drugs directly to the consumer (3). Nevertheless, representatives of pharmaceutical companies continue to provide doctors with a variety of "small gifts" (ranging from pens, notepads, calendars, to laser printers, bags, and decorative accessories) in order to ensure that their company's medications remain foremost in the physician's mind. Thus, whenever doctors are in office, they are surrounded by these marketing gifts, and are hence unknowingly exposed to implicit and explicit advertising.

Many ethical dangers may arise when physicians' prescribing behaviours are at risk of being unduly and disproportionately influenced by pharmaceutical companies (4). This is especially so when many doctors themselves indicate that their prescribing behaviours are influenced by their interactions with pharmaceutical companies and their representatives $(5,6)$. It may be argued that such marketing is even more dangerous than estimated, since many physicians think that their prescribing practices are not influenced by pharmaceutical companies even though they do accept the contact and their gifts (7).

The study aims to evaluate an important aspect of marketing of pharmaceutical products to physicians. We do this by quantifying so-called "minor gifts" present in physicians' offices in various medical care contexts. Furthermore, we 
compare whether any differences exist between the offices of psychiatrists and internal medicine physicians. We hypothesise that since psychiatrists treat patients with often chronic disorders, they may be subjected to more intense marketing by pharmaceutical companies. It may be suggested that the greater the presence of pharma branded material in the office, the more likely the doctor is to choose these medications. Thus, there would be an increased likelihood that their professional judgement is biased in some manner towards selecting those products for patient care.

\section{Methods}

This study examines variations in the number and type of minor gifts from pharmaceutical companies present in the offices of psychiatrists and internists in various medical contexts in Israel.

\section{Participants}

The offices of 74 (representing the total number of rooms available in three hospitals in the selected wards or outpatient clinics investigated) senior physicians, who do not share their rooms with non-physicians, were surveyed. Hospitals and physicians were selected by means of convenience sampling based on whether they agreed to participate in the study. These physicians' rooms were situated in four contexts (inpatient wards in two different psychiatry hospitals, one outpatient department and one general medical hospital) Thus, three hospitals were surveyed in total-Psychiatry Hospital A (inpatient and outpatient), Psychiatry Hospital B (inpatient wards only) and a General Hospital. The study was approved by the Helsinki Committee for Research at the Beer Yaakov Mental Health Center.

\section{Study procedure}

After explaining the purpose of the study and obtaining written approval from the physicians, the study team immediately entered the room of the physician (thus avoiding the risk of anyone removing or otherwise altering the presence of small pharma gifts). Each item bearing the name of a pharmaceutical product or company was documented and quantified. Information regarding the type of product, and how many such products branded with the names of pharmaceutical products or companies existed in each physician's room, was listed. This procedure was repeated in physicians' rooms in several medical care contexts, including physicians' offices in two psychiatry hospitals, one psychiatry outpatient department and one general medical hospital. All physicians' rooms in each hospital ward or clinic studied were investigated.

\section{Study analysis}

A general count was made of the number of products in each physician's rooms. A Kolmogorov-Smirnov test applied on the count of gifts indicated that it was not normally distributed $(p<0.001)$. Therefore, hypotheses testing was done using nonparametric tests. Comparisons were made between inpatient and outpatient psychiatry wards, as well as between the psychiatric and general medical hospitals.

\section{Results}

A total of 74 physicians' rooms, distributed among the two psychiatry hospitals (one of which had both inpatient and outpatient physicians' rooms investigated) and a general medical hospital, were sampled for the study (Table 1).

\begin{tabular}{|l|c|c|}
\hline \multicolumn{3}{|c|}{$\begin{array}{c}\text { Table 1 } \\
\text { Number of physicians' rooms investigated in } \\
\text { each medical care context }\end{array}$} \\
\hline & $\%$ & Number of rooms \\
\hline Psychiatry Hospital A & 18.9 & 14 \\
\hline Psychiatry Hospital B & 66.2 & 49 \\
\hline General medical hospital & 14.9 & 11 \\
\hline Total & 100 & 74 \\
\hline
\end{tabular}

Overall, a total of 687 gifts were counted in the physicians' rooms, with an average of 9.3 gifts per room (median $=7.0 ; \mathrm{SD}$ $=6.3$ ). The most common gift was notepads $(\mathrm{n}=150$, mean $=$ $2.0, \mathrm{SD}=2.0)$, followed by calendars $(\mathrm{n}=73$, mean $=1.0, \mathrm{SD}=$ 1.1 ) and medication guides ( $n=72$, mean $=1.0, S D=1.5$ ). The number of gifts sampled in the various physician rooms is shown in Table 2.

\begin{tabular}{|l|c|c|c|c|}
\hline $\begin{array}{c}\text { Table } 2 \\
\text { Mean, standard deviations, median and inter-quartile ranges of } \\
\text { number of advertising products according to distribution amongst } \\
\text { medical care contexts }\end{array}$ \\
\hline & Mean & $\begin{array}{l}\text { Standard } \\
\text { deviation }\end{array}$ & Median & $\begin{array}{l}\text { Inter quartile } \\
\text { range }\end{array}$ \\
\hline Psychiatry hospital A & 9.1 & 6.0 & 8.5 & 8.3 \\
\hline Psychiatry hospital B & 10.3 & 6.6 & 9.0 & 10.5 \\
\hline General medical hospital & 5.1 & 2.5 & 5.0 & 4.0 \\
\hline
\end{tabular}

As can be noted in Table 2, while there was no difference in the number of gifts counted in each physician's room between the two psychiatry hospitals ( $p=0.45$ ), more gifts were present in the psychiatric settings compared to the general medical hospital. A Mann-Whitney test for independent samples indicated a significant difference between the psychiatric hospitals and general medical hospital $(U=179, p=0.01)$. The difference between psychiatry inpatient $($ mean $=10.4$, $\mathrm{SD}=6.6)$ and outpatient wards $(M=9.0, \mathrm{SD}=6.1)$ was not significant $(p=0.48)$.

\section{Discussion}

Study observations indicate that the number of pharmaceutical company "minor gifts" in physicians' rooms is considerable. In addition, the study findings showed that the number of these gifts in psychiatric physicians' rooms was significantly higher than in general medical hospital physicians' rooms, indicating that the psychiatric setting is more "pharmafriendly".

There are several explanations as to why there appears to be more investment in gifts to psychiatrists than general physicians. First, many people tend to consider mental disorders as fundamentally different from physical disorders (8). It may be suggested that this may include seeing mental 
illness as more chronic than physical illness. Therefore, pharmaceutical companies may invest more intensively in marketing their products in psychiatric hospitals, as the medication will have to be taken over a longer period of time. Moreover, psychiatric patients start taking medications at a younger age and for a longer period of time. For example, an eighteen-year-old patient with recently diagnosed schizophrenia, may require antipsychotic medication for the rest of his life. This would be reflect a lucrative opportunity for pharma companies. Second, individuals who suffer from mental illness may be more likely to accept their doctors' recommended treatment since trust of the physician in psychiatric management is especially emphasised in the clinical relationship-once again potentially leading to a long-term use of the medication often for chronic conditions. Third, as there was no significant difference between the wards and the outpatient clinics with respect to the number of gifts provided to treating psychiatrists, it could be that pharmaceutical companies see a wide range of use for their medications in both inpatient and outpatient contexts as in the treatment of a range of conditions. Fourth, since in psychiatry there are many medications that have a similar mechanism of action (such as selective serotonin reuptake inhibitors [SSRIs]), and many medications may be given offlabel for various "dimensional" clinical conditions (for example antipsychotic medication for affective and anti-anxiety augmentation) (9), doctors may pick one medication over another based on their subjective or personal preference and experience. Thus, pharma companies may market more aggressively to psychiatrists in order to increase the chances of their products being prescribed.

Pharmaceutical companies would not invest considerable effort and resources in these minor gifts and their distribution if they did not believe that such efforts were not profitable for them. However, physicians may not be aware of how these so-called "minor gifts" affect their judgement and prescribing behaviours (10). A relatively recent systematic review on the subject strongly suggests that the interactions between physicians and the pharmaceutical industry and their marketing representatives, and the provision of gifts to doctors, do influence physicians' prescribing behaviour. Gifts, even those of low value, can influence the behaviour of those receiving the gift (11). Thus, such marketing strategies may encourage "irrational prescribing" based on physician bias in medication selection.

Moreover, the presence of these "small gifts" in the physicians' room may influence patients' perception of the physician. Many physicians think that they cannot be influenced by gifts (7). However, when physicians accept such gifts, they are perceived to offer lower quality care (12). In addition, a study of outpatients in a large medical centre in the US showed that most patients demonstrated less trust in their doctor if they knew that they had accepted gifts. Some patients stated that they would be less inclined to accept a prescribed medication if their doctor had been the beneficiary of any gift as a benefit of hearing a pharmaceutical representative's demonstration of the medication (13). Patients may meet their physician many times during the course of treatment interaction. This decisionmaking of the doctor and patient regarding treatment should be based on ethical principles and values without the extraneous influence of pharmaceutical companies (14). The danger thus exists that pharmaceutical companies which provide free gifts as part of their marketing strategy put economic priorities before the welfare of the patient and unduly influence the professional judgement of physicians.

This study sheds light on the phenomenon of offering physicians "small gifts" and demonstrates medical staff's level of exposure to this marketing material. "Small gifts" may lead to "irrational prescribing" of the pharmaceutical company's product. It is important to emphasise to clinical treatment staff the importance of maintaining professional judgement when managing patients and to highlight the potential dangers of exposure to ubiquitous advertising products. Much of the work of a doctor is maintaining their professional ethics and avoiding marketing strategies, economic pressure and other extraneous considerations when making decisions regarding their patients' wellbeing. The authors recommend policy development and education about the dangers of the phenomenon (15).

\section{Limitations of the study}

One of the limitations of this study is that the investigation did not examine several general hospitals and only focussed on one general hospital, because of which the results cannot be extrapolated to all general hospitals. In addition, in future studies, it would be interesting to compare the differences between other medical subspecialty departments in general hospitals.

\section{Conclusion}

To test the above hypotheses, it is necessary to perform further research to examine whether there is indeed a psychological effect or an impact on prescribing behaviour among physicians due to exposure from advertising. Thus, further studies should examine whether there is any association between the number of gifts in the room and the actual prescribing behaviour of the individual physician (specifically with regard to medications that are manufactured by the company whose gifts have been found in the physician's office). These studies should include mental healthcare providers since it appears that psychiatrists, at least from the findings of this small study, may receive more minor gifts. Continued research on the subject can shed more light on the impact of such marketing strategies in the healthcare system in general and mental health in particular. In addition, it will also be interesting to examine differences in the effect of exposure advertising targeting doctors in Israel and other countries. The medical profession should strive to attain objectivity in clinical management. Awareness of any interfering factor, while maintaining physician autonomy, should be encouraged and maintained. 


\section{Conflicts of Interests and funding: None}

\section{Acknowledgements: None}

References

1. King M, Bearman PS. Gifts and influence: Conflict of interest policies and prescribing of psychotropic medications in the United States. Soc Sci Med. 2017 Jan; 172: 153-62.

2. Linder R. Marketing machine: Pharmaceutical companies in Israel invest more than 100 million dollars a year in marketing to about 1510 thousand doctors. Themarker.com. 2004 Apr [cited 2018 Dec 29]. Available from https://www.themarker.com/consumer/1.479194

3. Israel Ministry of Health [Internet]. Special Directive number 13/2018. 2018 Oct 22[cited 2018 Dec 29]. Available from https://www.health. gov.il/hozer/mk13_2018.pdf

4. Marco CA, Moskop JC, Solomon RC, Geiderman JM, Larkin GL. Gifts to physicians from the pharmaceutical industry: an ethical analysis. Ann Emerg Med. 2006 Nov; 48(5): 513-21.

5. Brett AS, Burr W, Moloo J. Are gifts from pharmaceutical companies ethically problematic? Arch Intern Med. 2003 Oct 13; 163(18): 2213-18.

6. Roy N.Who rules the great Indian drug bazaar? Indian JMed Ethics. 2004 Jan-Mar;1(1):2-3.

7. Salmasi S, Ming LC, Khan TM. Interaction and medical inducement between pharmaceutical representatives and physicians: a metasynthesis.J Pharm Policy Pract. 2016 Nov 17; 9: 37.

8. Kendell RE. The distinction between mental and physical illness. $\mathrm{Br} J$ Psychiatry. 2001 June; 178: 490-493.

9. Chen J, Gao K, Kemp DE. Second-generation antipsychotics in major depressive disorder: update and clinical perspective. Curr Opin Psychiatry. 2011 Jan; 24(1):10-7.

10. Gold A, Appelbaum PS. Unconscious conflict of interest: a Jewish perspective.J Med Ethics. 2011 July; 37(7): 402-05.

11. Macneill PU, Kerridge IH, Newby D, Stokes BJ, Doran E, Henry DA Attitudes of physicians and public to pharmaceutical industry 'gifts'. Intern Med J. 2010 May; 40(5):335-41.

12. Lee $D$, Begley CE. Physician report of industry gifts and quality of care. Health Care Manage Rev. 2016 July 1, 41(3): 275-8.3

13. Green MJ1, Masters R, James B, Simmons B, Lehman E. Do gifts from the pharmaceutical industry affect trust in physicians? Fam Med. 2012 May; 44(5):325-31.

14. Bansal RK, Das S. Unethical relationship between doctors and drug companies. J Ind Acad Forensic Med. 2005 Jan; 27(1): 40-2.

15. Fickweiler F, Fickweiler W, Urbach E. Interactions between physicians and the pharmaceutical industry generally and sales representatives specifically and their association with physicians' attitudes and prescribing habits: a systematic review. BMJ Open. 2017 Sept 27; 7(9): e016408.

\section{Assessing completion reports for compliance with institutional ethics committee-approved protocols: An observational study.}

\section{SNEHALATA V GAJBHIYE, SHARMILA V JALGAONKAR, SARITA G DABBA, SHWETA V SURVE, MANASI S LAD}

\section{Abstract}

Background: Protocol non-compliance in clinical research studies is common and can affect both patient safety and data integrity. There are no published studies which actively looked for non-compliance. The present study was carried out, against this background, with the objective of assessing the proportion of protocol non-compliance and evaluating those aspects of protocol where there was non-compliance.

Methods: The study completion reports that were submitted to

Authors: Snehalata V Gajbhiye (dr.ssborkar@gmail.com), Assistant Professor, Department of Pharmacology and Therapeutics, Seth GS Medical College and KEM Hospital, Parel, Mumbai 400012 INDIA; Sharmila V Jalgaonkar (corresponding author-sharmila_jalgaonkar@rediffmail.com) Sarita G Dabba (saritadabba99@gmail.com), Senior Executive, Medical Services. SIRO Clinpharm Pvt. Ltd. Wagle Industrial Estate, Thane West, Thane, 400604 INDIA; Shweta Surve (shwetavsurve2@gmail.com), Executive Assistant, Institutional Ethics Committee, Seth GS Medical College and KEM Hospital, Parel, Mumbai 400012 INDIA; Manasi S Lad (manasilad0@gmail. com), Executive Assistant, Institutional Ethics Committee, Seth GS Medical College and KEM Hospital, Parel, Mumbai 400012 INDIA

To cite: Gajbhiye SV, Jalgaonkar SV, Dabba SG, Surve SV, Lad MS. Assessing completion reports for compliance with institutional ethics committeeapproved protocols: An observational study. Indian J Med Ethics. 2020 AprJun;5(2) NS: 119-23.DOI:10.20529/JMME.2020.025.

Published online on March 2, 2020.

Manuscript Editor:Vijayaprasad Gopichandran

Peer Reviewers: S Swarnalakshmi and an anonymous reviewer

OIndian Journal of Medical Ethics 2020 the institutional ethics committee for the period January 2017 to December 2017 were compared with the approved protocol. A checklist for recording protocol non-compliance was developed, which was validated by five experts and consisted of a 12-point checklist with responses such as yes, no, not applicable, and insufficient information.

Results: Out of 193 studies, prospective observational studies were $n=120(62.17 \%)$, retrospective studies were $n=39(20.21 \%)$, interventional studies $n=28(14.51 \%)$, and observational studies with both prospective and retrospective study design were $n=6$ (3.11\%). The study objective was modified in $n=18(9.32 \%)$ studies. Only $n=14$ (7.24\%) satisfied the selection criteria. Six studies (3.10\%) did not collect the data as mentioned in the protocol. Fifty-eight studies (30.05\%) did not achieve the calculated sample size, whereas $n=78(40.41 \%)$ did not complete the study as per the stipulated study duration. Contrary to 180 protocol deviations found in this study, only 14 protocol deviations were reported by the principal investigator. Aspects like blinding and randomisation, which are relevant to interventional studies $(n=$ 28), showed $100 \%$ compliance.

Conclusion: The research protocol is not adhered to in all aspects. Adequate training to investigators will help prevent noncompliance and enable us to conduct studies with higher ethical and scientific integrity.

Keywords: study design, sample size, interventional studies, noncompliance 